\title{
A new-age Hooke book
}

Focus on Multidimensional Microscopy Volumes 1 and 2

edited by P. C. Cheng, P. P. H wang, J. L. Wu, G. Wang and H. Kim

World Scientific Publishers · December 1999

Volume $1-$ Hardback $£ 57 / \$ 91$

Volume 2 - Hardback $£ 64 / \$ 103$

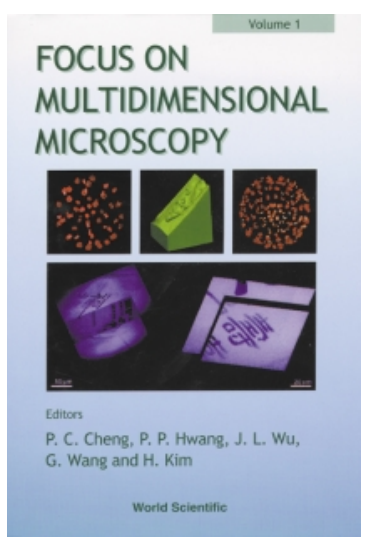

Warren Zipfel

$\mathrm{M}$ icroscopy, along with virtually everything else, recently entered the 21 st century. The past few decades produced some remarkable innovations in light microscopy - Nomarski optics, video microscopy, confocal microscopy, multiphoton microscopy and deconvolution methods were all made possible by a combination of ingenious thinking, modern electronics, lasers and fast computers. Genomics and proteonomics are now the hot topics and, hopefully, we will soon be talking about 'physionomics', where genes and their associated products will be better understood at the level of cell biology and functional physiology. The characteristics of these new microscopies - high contrast, sensitive detection, 3D sectioning - are precisely what is needed to obtain the dynamic live-cell and in vivo information that will help to link gene and gene product to cellular function. With this perspective I examined the two volumes of Focus on Multidimensional M icroscopy, a collection of chapters devoted to modern microscopy with, as the editors say in the preface, emphasis on "multidimensional and multimodality microscopy". The term multidimensional microcopy most commonly refers to $3 D$ or $4 D$ (three spatial dimensions measured over time) image data and is usually equated with the dynamic imaging of function and morphology.

The preface also tells us that the two volumes are arranged with coverage of various forms of modern microscopy in the first volume, and chapters demonstrating "typical microscopical applications" in the second. However, chapters covering applications and those dealing with microscope technology seem to be about evenly divided between the two volumes. $M$ any of the chapters covering applications simply involve the use of conventional light or electron microscopy to investigate a particular biological question, and are devoid of specific details concerning the microscopy used. Although they may cover important work in their respective areas of biology, they seem out of placein the context of the two books as they do not relate directly to the technologies presented in the other sections.

The chapters covering new microscope technologies and techniques are typically short, but well written. They are, however, very specialized, and many are written by leading experts in microscopy and optics. This is particularly true of the five chapters by Colin Sheppard and co-authors, covering topics ranging from the description of a scanned-optical-fibre reflection microscope to calculations of the optical-transfer function of a 4-Pi confocal microscope. Other topics include near-field microscopy and spectroscopy of single molecules by Sunney Xie and colleagues. Unlike many of the other chapters, this section is especially thorough and has a detailed reference list, making it a good introduction to a field that should find many future applications. Some of the contributions are relatively short, but focus on a fundamental concept of importance to all microscopists. The best example is Jim Pawley's brief chapter on resolution and photon statistics in confocal microscopy. Those involved with Pawley's annual 3D-microscopy course know his fervor on this subject. The effective 'resolution' achieved in most experiments involving living specimens is limited by the number of photons that can be collected before damaging the sample, rather then by bumping up against the diffraction limit. Light is often not as benign as we would like it to be.

The preface tells us that the technical chapters cover areas such as multiphoton microscopy, confocal microscopy and X-ray microscopy, but for the most part they only deal with specific aspects of these subjects, rather than giving overviews of the particular technology. For example, there is a chapter comparing confocal and multiphoton imaging in scattering samples. Researchers working with thick specimens such as tissue explants or thoseattempting in vivo imaging who are al ready familiar with these forms of 3D microscopy would benefit from this chapter, but a newcomer to the field would get very little out of it. The two volumes are definitely not an introduction to the modern incarnations of microscopy, but more a series of research papers, either on specific topics within these incarnations, or demonstrating new forms that might prove useful in the future. Many chapters contain detailed mathematical coverage of their particular topics and the two volumes are probably better suited for researchers who are interested in microscope development rather than practising cell biologists or physiologists looking for an introduction to the newer forms of microscopy.

Warren Zipfel is in the Developmental Resource for Biophysical Imaging and O ptoElectronics, Cornell University, Ithaca, New York 14853, USA email:wrz2@cornell.edu

\section{Other microscopy books Hardback}

Confocal Microscopy Methods and Protocols

edited by Stephen W. Paddock Humana Press, $€ 69 / \$ 99.50$

Three-Dimensional Electron Microscopy of Macromolecular Assemblies by Joachim Frank Academic Press, $£ 75 / \$ 99$

Working with the Scanning Electron Microscope

by S. K. Chapman Lodgemark, £9.95/\$15

Early History of the Electron Microscope by L. Marton San Francisco Press, $£ 10 / \$ 15$

Principles and Techniques of Electron Microscopy: Biological Applications edited by M. Hayat

Cambridge University Press, $€ 65 / \$ 100$

Continued on pE223 